\title{
Scanning organized material: Individual differences in search strategies
}

\author{
CHARLES I. MANISCALCO and DONALD V. DeROSA \\ Bowling Green State University, Bowling Green, Ohio 43403
}

\begin{abstract}
A recognition memory experiment investigated memory scanning of material with varying degrees of organization. Subjects were shown a series of three, four, or five digits (positive set) followed by a probe stimulus in a paradigm developed by Sternberg (1966). The results indicated that, for unorganized positive sets, all subjects performed a serial search, with reaction time increasing across set size. For highly organized sets, some subjects continued to perform a serial search, while others engaged in a unit search, evidenced by the fact that reaction time was not related to set size. These two groups also differed in their overall response latencies, and a signal detection theory analysis indicated that subjects choosing a serial strategy were more cautious (i.e., more concerned with accuracy) than subjects choosing a unit strategy.
\end{abstract}

Sternberg (1966) introduced a paradigm for studying retrieval processes in a recognition memory experiment. Since that time, most of the research has supported the notion that memory search is both serial and exhaustive (see Sternberg, 1975, for a review). Yet, support has not been universal. One noteworthy exception involves studies examining the effects of stimulus organization on memory search (Baumgarte \& DeRosa, 1973; DeRosa \& Beckwith, 1971; DeRosa \& Tkacz, 1976). DeRosa and Tkacz (1976), for example, used stimuli which were not easily labeled verbally but which could be organized into units. Specifically, the stimulus sets comprised ordered sets of nine items (pictures of action, e.g., a bird in flight). Memory sets of three, four, or five items were constructed from these stimuli, with some sets composed of a consecutive sequence of items (closed sets) and others composed of a nonconsecutive sequence of items (open sets). When reaction time (RT) was plotted as a function of set size, the typical set size effect was present for open sets, replicating Sternberg's (1966) findings. For closed sets, however, the slope of the curve was essentially zero, indicating a parallel search strategy.

DeRosa and Tkacz (1976) proposed that highly organized stimuli (i.e., closed sets) may allow the set to be processed as a unified whole; subsequently, probes could be compared to the unit rather than to each individual positive set element. Regardless of set size, the subject's decision is always the result of a comparison with a unified whole, and set size should not influence RT. The question remained, however, of whether or not this unit search strategy is generalizable beyond "nonverbal" stimuli. One of the purposes of the present study, then, was to determine if the use of highly organized sets of digits results in a unit search strategy.

This study was supported by NSF Grant BG-16729 awarded to the second author. Requests for reprints should be sent to Donald V. DeRosa, Department of Psychology, Bowling Green State University, Bowling Green, Ohio 43403.

\section{METHOD}

Subjects

The subjects were 12 undergraduate students enrolled at Bowling Green State University, and each participated in two 1-h sessions.

\section{Stimuli and Apparatus}

Subjects were individually tested in a sound-deadened isolation chamber, with the digits 1 through 9 presented visually on an I.E.E. rear-projection display cell mounted at eye level $3 \mathrm{ft}$ in front of the subjects. The subjects' RTs, measured by a Hunter Klockounter to the nearest $.001 \mathrm{sec}$, were recorded on punched paper tape for summary by computer. Two lights mounted on the display panel signaled correct and incorrect responses for accuracy feedback.

\section{Procedure}

Each trial was preceded by a warning signal, which consisted of an asterisk displayed on the viewing face of the cell for $300 \mathrm{msec}$. The signal was followed by a serial presentation of three, four, or five digits (positive set), with each digit on for $300 \mathrm{msec}$ and a 300 -msec off-time between digits. One second after the presentation of the positive set, another warning signal (an amber light centered directly above the display cell) appeared until a single digit (probe) was presented $2 \mathrm{sec}$ later. Subjects were instructed to depress a key marked "yes" if the probe was identical to any member of the positive set and to depress a key marked "no" otherwise.

\section{Experimental Conditions}

Subjects received $1 / 2 \mathrm{~h}$ of practice trials and 576 experimental trials during the two sessions. Each subject participated in all conditions. The types of positive sets were the same as those used by DeRosa and Beckwith (1971), and the same terminology is used. A closed set is one in which the elements can be permuted so as to be consecutive (e.g., 4, 3, 5, 6). The elements of an open set cannot be permuted into a consecutive sequence (e.g., 1, 4, 3, 6). The sequence in which the elements of the positive sets are shown further distinguishes the positive sets. In an increasing set, the elements are presented in ascending order (e.g., 5, 6, 7, 8); in an irregular set, the elements are presented in random order with consecutive runs no greater than two. The resulting types of positive sets are open-irregular, closed-irregular, and closed-increasing. Presentation of the positive sets to the subjects was blocked into sets of 192 trials each, according to the above classification, with rest intervals both within and between the three different blocks. The order of presentation 
was determined randomly for each subject, with each of the six possible orderings of the three blocks occurring twice over all subjects.

Probe items were identical to items in each serial position with equal frequency. Additionally, the probability over all trials that a probe was identical to an element of the positive set was .5. Following completion of the experimental trials, subjects were debriefed and reported their strategies on a short questionnaire.

\section{RESULTS AND DISCUSSION}

\section{Set Size Data}

Mean RTs for correct responses were computed for each subject. The error rates averaged $3.3 \%$ and ranged from $1.7 \%$ to $6.6 \%$. Figure 1 shows the RT data for positive and negative responses as a function of set size, with each panel representing one type of organization (open-irregular, closed-irregular, and closed-increasing sets).

The RT functions for the open-irregular sets (left panel of Figure 1) show that the typical increasing set size functions were observed, with set size being a highly significant variable $[F(2,22)=18.14, p<.001]$. More important, both functions increased at approximately the same rate. The equations describing the best-fitting straight lines yielded a slope of $28 \mathrm{msec}$ per unit increase in set size for positive responses; the comparable figure for negative responses was $30 \mathrm{msec}$. Consequently, there was no significant Type of Response by Set Size interaction. The linear increase in RT, along with the lack of an interaction, can be taken as support of Sternberg's serial exhaustive model of memory search.

The other panels in Figure 1 represent data obtained when positive sets had greater organization. Both panels show a pattern of results different from that obtained when open sets were used. Three of the four curves for the closed sets (middle and right panels) show marked reductions in slope. For closed-irregular sets (middle

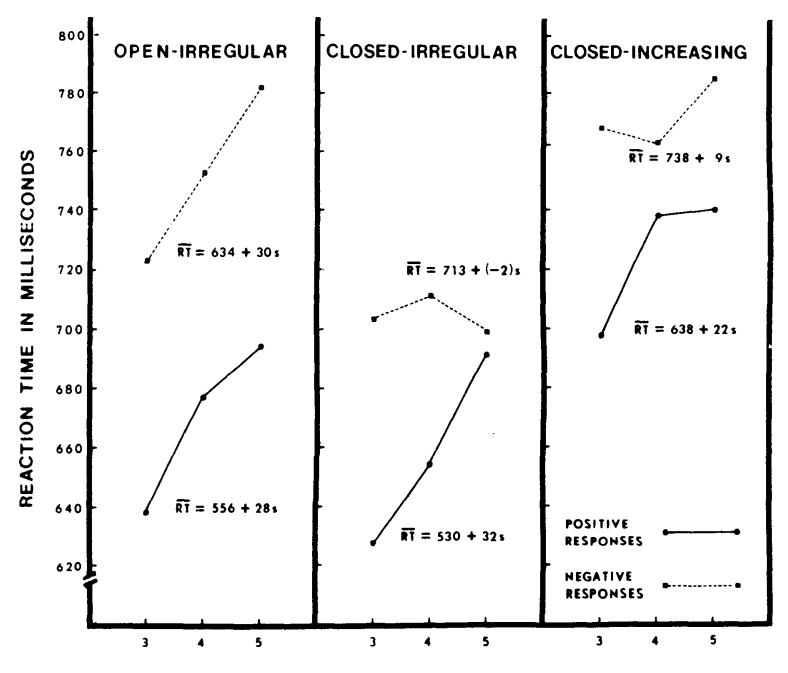

SET SIZE

Figure 1. Reaction time as a function of set size for the three types of sets. Equations describing the best-fitting (by least squares) linear functions are provided. panel), negative responses resulted in a flat function and positive responses continued to increase with set size, resulting in a significant Type of Response by Set Size interaction $[F(2,22)=13.74, p<.001]$. Data for the closed-increasing sets (right panel) show a reduction in slope compared to the curves for open sets, yet set size remained a marginally significant variable $[F(2,22)=$ $3.61, \mathrm{p}<.05$ ]

Assume that utilizing the organization of the positive sets is a voluntary decision by the subject. It is then possible that the functions for the closed-increasing sets represent a combination of strategies, with some subjects using the organization present in the positive sets, and perhaps comparing the probe to a single unit, and others using a serial search. In fact, 6 of the 12 subjects reported that, for the closed-increasing sets, they tried to remember the first and last items. When the probe appeared, they responded "yes" if it was within the region defined by these items and "no" if it was outside the region. Considering these reports, it is not surprising that the average functions for closed-increasing sets are difficult to interpret.

In an effort to clarify interpretations, subjects were divided on the basis of their reports. Figure 2 shows RT functions for those subjects not reporting a parallel strategy [serial search subjects (S)]; data for those subjects who did so [unit search subjects (U)] are shown in Figure 3.

The functions for the open-irregular sets for both groups of subjects have the same form as the functions in Figure 1. Set size was a significant variable for both $\mathrm{S}$ subjects $[F(2,10)=9.13, p<.01]$ and $U$ subjects $[F(2,10)=9.45, p<.01]$. Only the linear component of set size was significant in both cases. The functions for the closed-irregular sets also are similar to those in Figure 1. For $\mathrm{S}$ and $\mathrm{U}$ subjects, there was no significant set size effect but a significant interaction of Set Size by Response Type $[F(2,10)=15.17, \mathrm{p}<.001$, for $\mathrm{S}$ subjects and $F(2,10)=7.12, p<.05$, for $U$ subjects]. This interaction, as in Figure 1, was due to essentially flat functions for negative probes and increasing functions for positive probes. These analyses indicate that both groups dealt with open-irregular and closed-irregular sets in a similar manner. The functions for the closedincreasing sets present a different picture. The slopes of the best-fitting lines for $\mathrm{S}$ subjects were 40 and $22 \mathrm{msec}$ for positive and negative responses, respectively. For the U subjects, the slopes were 3 and -4 msec for "yes" and "no" responses. The analyses yielded a significant set size effect for $S$ subjects $[F(2,10)=9.70, p<.01]$. Only the linear component of set size was significant $[F(1,5)=11.10, p<.05]$. The set size variable was not significant for the $U$ subjects.

Dividing subjects on the basis of introspective reports must be viewed with caution. Doing so, however, yielded some dramatic differences in the observed data. First, it seems that all subjects dealt with open-irregular and closed-irregular sets in the same manner. However, for closed-increasing sets, some subjects used a unit search 


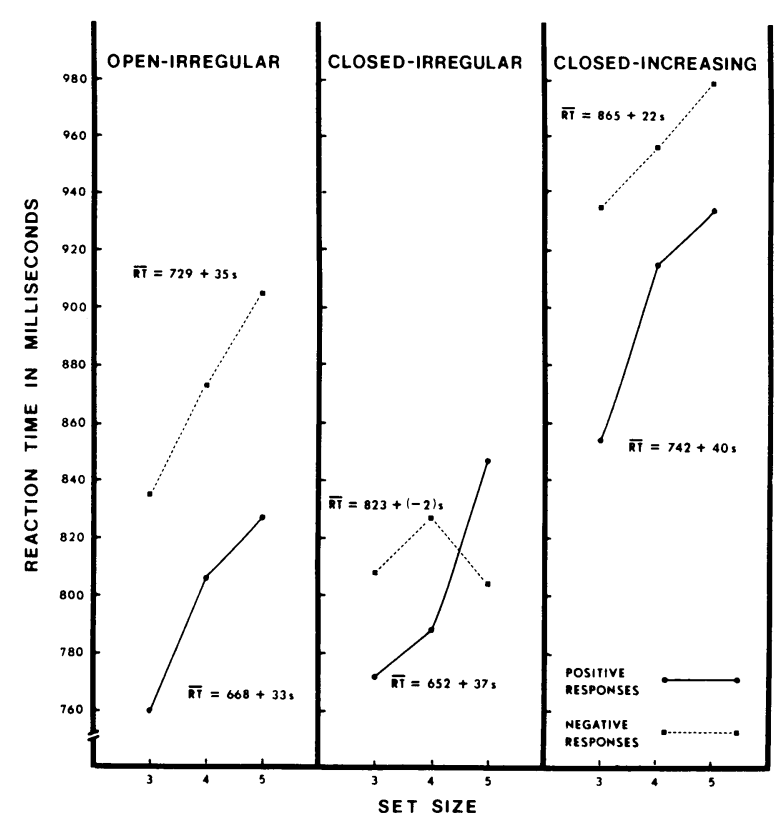

Figure 2. Reaction time as a function of set size for the three types of sets: $\mathrm{S}$ subjects.

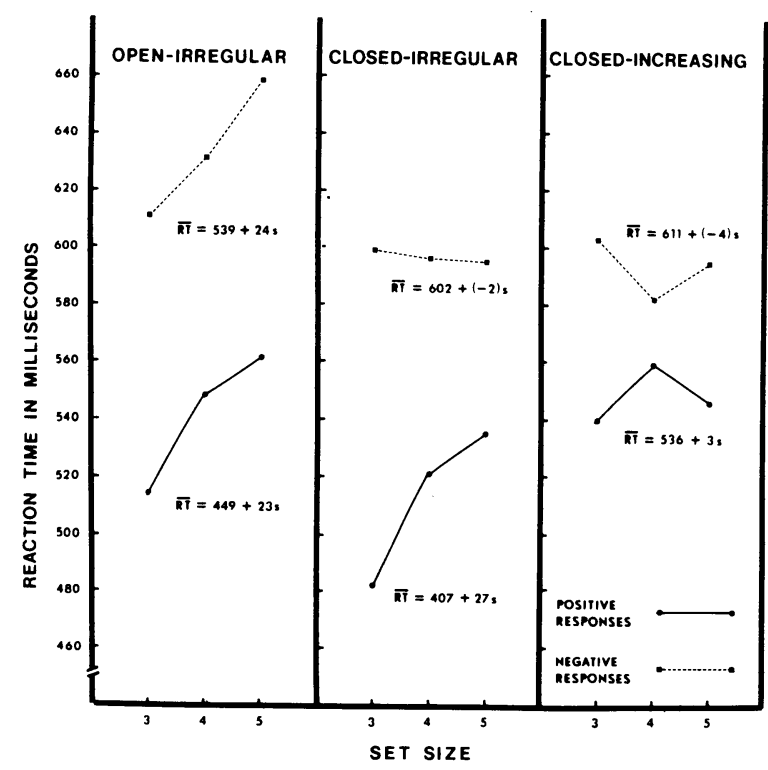

Figure 3. Reaction time as a function of set size for the three types of sets: $U$ subjects.

(as evidenced by near-zero slopes) and others used a serial search. Clearly, subjects do have the ability to utilize the organization present in the to-beremembered sets, allowing for a choice of search strategy. That not all subjects did so supports the notion that this is a voluntary process in much the same manner as the control processes described by Atkinson and Shiffrin (1968). Also, the division on the basis of introspective reports indicates that subjects could accurately reflect upon processes whose durations are on the order of milliseconds. A surprising result concerns the difference in vertical displacement of the functions for $\mathrm{S}$ and $\mathrm{U}$ subjects. The mean RT for $\mathrm{S}$ subjects was $860 \mathrm{msec}$, with individual means ranging from 699 to $1,010 \mathrm{msec}$; the RT for U subjects was $573 \mathrm{msec}$, ranging from 417 to $616 \mathrm{msec}$. Overall, then, $\mathrm{S}$ subjects were considerably slower than $U$ subjects in responding.

\section{Signal Detection Theory (SDT) Analysis}

The present data are nicely suited to a SDT analysis (Swets, 1973), and nonparametric measures of sensitivity $[\mathrm{P}(\mathrm{I})]$ and decision criterion $\left(\mathrm{B}^{\prime \prime}\right)$ were calculated for each subject (Grier, 1971). The mean P(I) values for $S$ and $U$ subjects (.970 and .965 , respectively) were not significantly different from one another $[\mathrm{t}(10)=.57$, $\mathrm{p}>.05]$. The mean $B^{\prime \prime}$ values $(.165$ and -.138 for $S$ and U subjects, respectively) were significantly different $[t(10)=1.91, p<.05]$. Thus, the two groups were not different in their perceptual sensitivity to the stimuli, but the $\mathrm{S}$ subjects had a more conservative decision criterion than the $U$ subjects.

Although differences in decision criteria between the two groups may not directly explain the overall RT and search strategy differences, they may reflect underlying motivational differences. The $\mathrm{U}$ subjects may have been more highly motivated than the $S$ subjects, resulting in faster RTs and the use of an efficient unit search strategy when appropriate.

\section{Remoteness Data}

DeRosa and Beckwith (1971) and DeRosa and Morin (1970) showed that RTs to negative probes were systematically related to the ordinal distance of the probe to the nearest member of the positive set. As probes became more remote (further from the nearest member of the positive set), negative reactions were faster. Figure 4 shows the remoteness functions for the present study. Remoteness was a highly significant variable $[F(3,33)=$ $40.88, \mathrm{p}<.001]$. Although the three functions were not significantly different from one another, the interaction of Remoteness by Type of Positive Set was significant $[F(6,66)=3.79, p<.01]$. Further, the interaction was significant only for the linear component of the remoteness variable $[\mathrm{F}(2,22)=10.33, \mathrm{p}<.001]$.

DeRosa and Beckwith (1971) postulated that negative items nearest to a well-defined region (such as that defined by a closed-increasing set) are more similar to elements in the positive set than those that are more remote. The inflated RTs for these items can be explained, then, by a response competition notion. That is, the binary decision of "yes-no" is more difficult for items nearer the positive set because of relatively greater similarity of these items to the items comprising the positive set. Closed-increasing sets define a region more clearly than do the other types of sets, and the consequence is relatively more response competition for negative items near this region.

To summarize, there is certainly evidence for the use of a unit search strategy for highly organized sets. Further, its use appears to represent a voluntary process. As such, it is unrealistic to expect all subjects to use a 


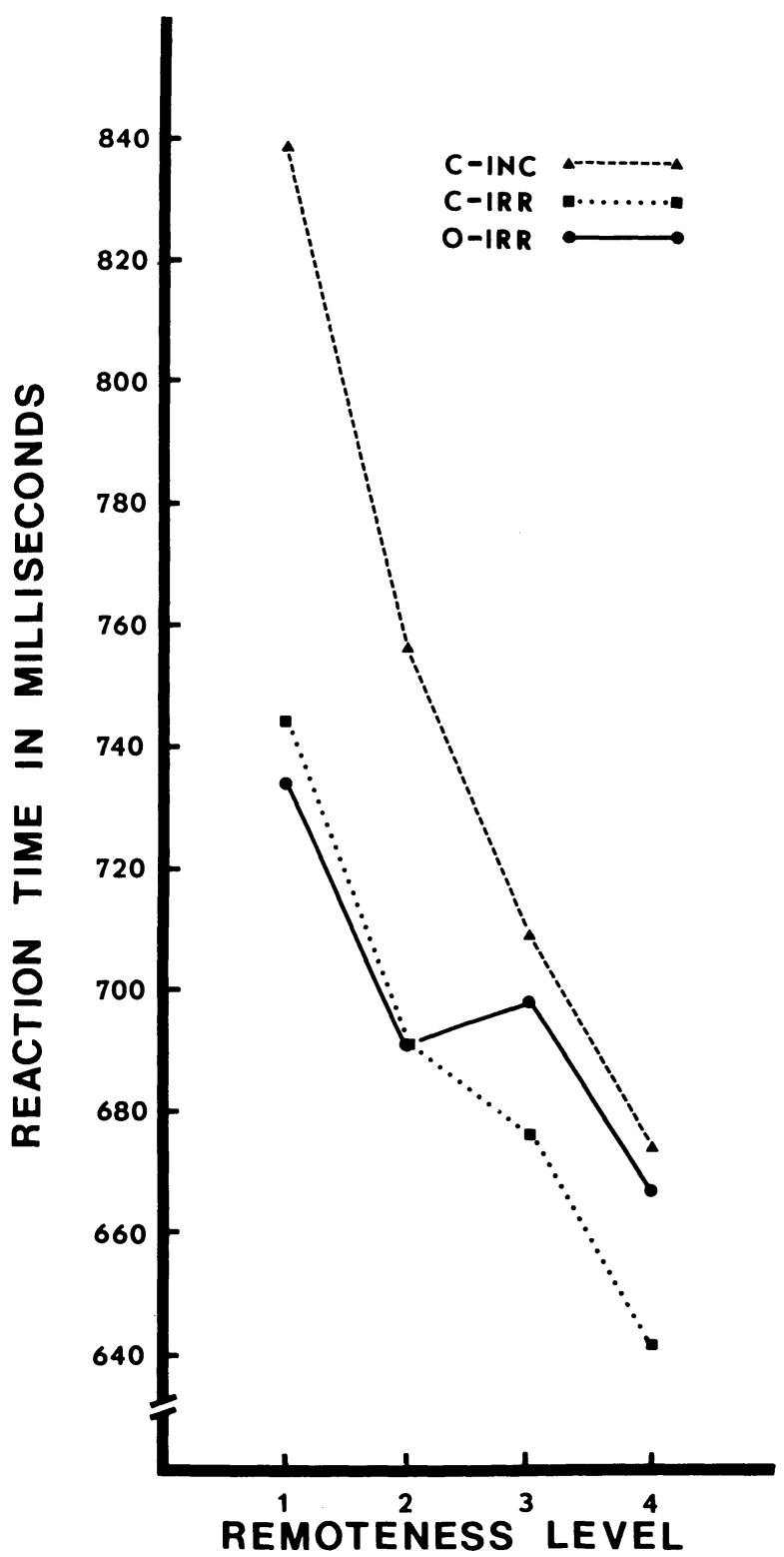

Figure 4. Reaction time for negative responses for each type of set as a function of remoteness. (O-IRR $=$ open-irregular, C-IRR = closed-irregular, $\mathrm{C}-\mathrm{INC}=$ closed-increasing.)

unit search in the presence of highly organized sets. The question then becomes: When, and by whom, will a unit search be used? The results of the SDT analysis suggest that the subject's relative concern for speed and accuracy may determine the type of search strategy used. Subjects concerned with speed may engage in a unit search for highly organized sets, whereas those interested in accuracy may continue to use a slower serial search. If this is so, it should be possible by manipulation of instructions and/or payoff matricies to alter the subject's preference for speed and accuracy and, consequently, to alter the type of search used. On the other hand, Hunt, Frost, and Lunneborg (1973) suggested that performance on a recognition memory task may be related to individual differences in verbal and spatial abilities. In future research, selection of subjects on the basis of these differences in cognitive abilities may aid in a better understanding of the relationship between subject characteristics and recognition memory performance.

\section{REFERENCES}

Atkinson, R. C., \& Shiffrin, R. M. Human memory: A proposed system and its control processes. In K. W. Spence \& J. T. Spence (Eds.), The psychology of learning and motivation: Advances in research and theory (Vol. 2). New York: Academic Press, 1968.

Baumgarte, R., \& DeRosa, D. V. Information processing in a two-item classification task: Relationships among items in a memorized set. Journal of Experimental Psychology, 1973, $100,1-7$.

DeRosa, D. V., \& Beckwith, M. Retrieval of information from organized memory sets. Psychonomic Science, 1971, 23, 177-179.

DeRosa, D. V., \& Morin, R. E. Recognition reaction time for digits in consecutive and nonconsecutive memorized sets. Journal of Experimental Psychology, 1970, 83, 472-479.

DeRosa, D. V., \& TKacz, S. Memory scanning of organized visual material. Journal of Experimental Psychology: Human Learning and Memory, 1976, 2, 688-694.

Grier, J. B. Nonparametric indexes for sensitivity and bias: Computing formulas. Psychological Bulletin, 1971, 75, 424-429.

Hunt, E. G., Frost, N., \& Lunneborg, C. L. Individual differences in cognition: A new approach to intelligence. In G. Bower (Ed.), Advances in learning and motivation (Vol. 7). New York: Academic Press, 1973.

Sternberg, S. High-speed scanning in human memory. Science, $1966,153,652-654$.

Sternberg, S. Memory scanning: New findings and current controversies. Quarterly Journal of Experimental Psychology, $1975,27,1-32$.

Swets, J. A. The relative operating characteristic in psychology. Science, 1973, 182, 990-1000.

(Received for publication August 15, 1979.) 